Near-Field Cosmology with Dwarf Elliptical Galaxies

Proceedings IAU Colloquium No. 198, 2005

(C) 2005 International Astronomical Union

B. Binggeli, H. Jerjen, eds. DOI: $00.0000 / \mathrm{X} 000000000000000 \mathrm{X}$

\title{
The stellar populations of local dwarfs
}

\author{
Enrico V. Held ${ }^{1}$ \\ ${ }^{1}$ Osservatorio Astronomico di Padova, INAF, vicolo dell'Osservatorio 5, I-35122 Padova, Italy
}

\begin{abstract}
Recent progress in our knowledge of stellar populations in local dwarf spheroidal galaxies is briefly discussed. A few results are summarized including wide field observations of stellar populations and their spatial variations, studies of AGB and variable stars, extension to near-infrared wavelengths, and the interpretation effort based on synthetic color-magnitude diagrams and chemical evolution models.
\end{abstract}

Keywords. galaxies: dwarf, Local Group, galaxies: stellar content, galaxies: evolution

\section{Introduction}

The study of stellar populations of nearby dwarf galaxies provides a powerful means of learning, albeit indirectly, about the evolution of low mass galaxies at large look-back times. Galaxies in the Local Group are close enough that they can be resolved in the constituent stars and studied using the classical tools of stellar population work, the HR diagram in the first place. Diagnostics derived from the color-magnitude diagram of local dwarf galaxies are used to pinpoint and, to some extent, quantify the presence of young, intermediate-age (1-9 Gyr), and old ( $\gtrsim 10 \mathrm{Gyr}$ ) stellar populations and to reconstruct their histories of star formation. Recent advances with spectroscopy of individual stars and nebulae provide complementary information to understand their chemical enrichment histories. I will focus here on the resolved stellar populations of dwarf spheroidal (dSph) galaxies, with the exclusion of nearest Milky Way companions and the dwarf galaxies around M31 and in nearby groups, which are the subject of other contributions to this conference. I refer the reader to existing reviews (Mateo 1998, Da Costa 1998 van den Bergh 2000, Grebel 2000, Tolstoy 2003, Gallagher \& Grebel 2004) for earlier work and a comprehensive account of stellar populations in Local Group dwarfs as emerged from the last decade of HST and ground-based studies.

\section{The stellar populations of dwarf spheroidal galaxies}

\subsection{Stellar populations: recent results}

Direct age measurements of the main-sequence turnoff in nearby dSph, as well as indirect evidence from horizontal branch stars in more distant dwarfs, have shown that all dwarf spheroidals in the Local Group are characterized by a common early epoch of star formation approximately coeval to the Galactic globular clusters (10-13 Gyr ago) (e.g., Held et al. 2000 Grebel 2000). This initial star formation episode is predominant in some dSph, but the general evidence is for multiple episodes or a continuous star formation until very recent times.

Two examples of the color-magnitude diagrams (CMDs) of "young" and "old" dwarf spheroidal galaxies are shown in Fig. 1 in both cases, the stellar populations show a complexity typical of a range of age and metallicity. Fornax (see Fig. 1 left panel; also 

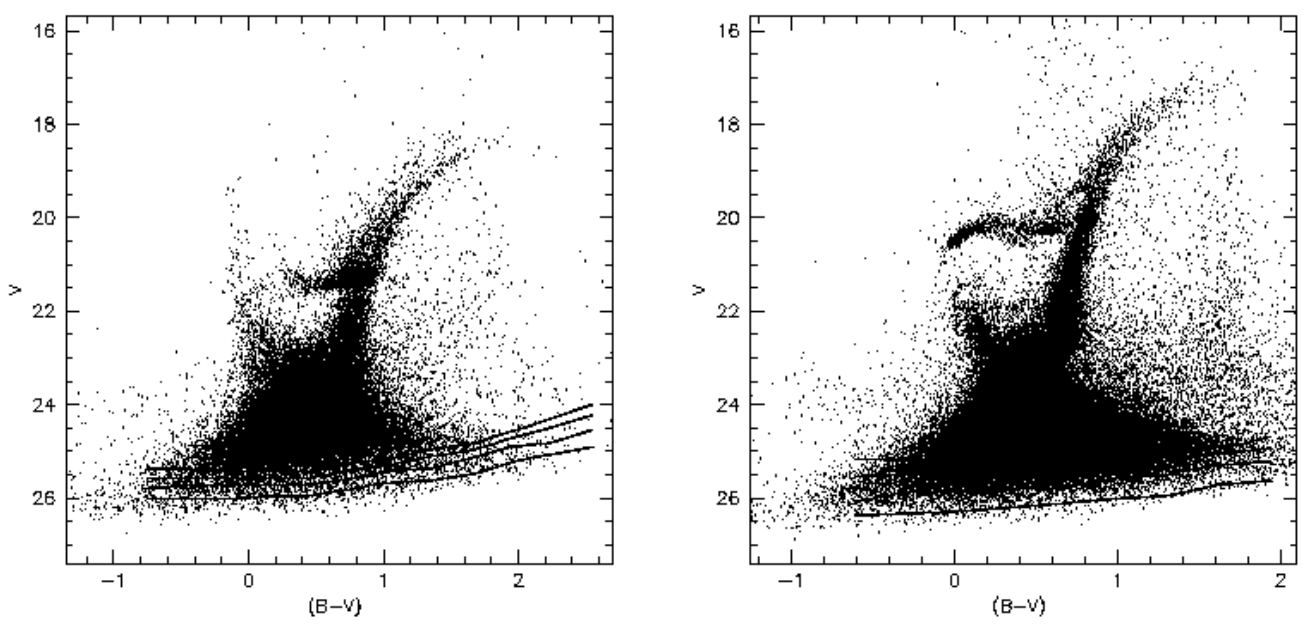

Figure 1. Wide-field color-magnitude diagrams of Fornax (left) and Sculptor (right) dwarf spheroidals based on ESO/WFI observations (Rizzi et al. 2005, in prep.).

Saviane et al. 2000) is an example of a "young" dwarf spheroidal: its CMD is characterized by a "blue plume" of main-sequence stars indicating an extended period of star formation, until a few hundred Myr ago. The very wide red giant branch clearly shows the presence of a mix of old and intermediate-age stars with different metal abundances. Pont et al. (2004) have recently studied the star formation and chemical enrichment in a central region of Fornax by combining VLT photometry and stellar spectroscopy. Their observations suggest an increase in star formation rate and metallicity in the last few Gyr: the metallicity distribution of Fornax is centered at $[\mathrm{Fe} / \mathrm{H}]=-0.9$ with a long tail of metal-poor stars extending to $[\mathrm{Fe} / \mathrm{H}]=-2.0$ and a number of stars as metal-rich as $[\mathrm{Fe} / \mathrm{H}]=-0.4$.

The Carina dwarf is another example of dSph galaxy with a prominent intermediateage population. With two main bursts occurring 5 and $11 \mathrm{Gyr}$ ago, and a small population of stars on a younger main sequence (up to 0.6 Gyr ago), Carina is the best example of an episodic star formation history (see Monelli et al. 2003 and refs. therein). This galaxy also provides the most dramatic example of an "age-metallicity degeneracy", i.e. the balance of competing and superposed effects of a younger age and an increasing metallicity on the CMD distribution of red giant stars. Yet its very narrow red giant branch, seemingly contrasting the multiple star formation episodes, does not imply a small range in $[\mathrm{Fe} / \mathrm{H}]$. A quantitative analysis of the CMD of Carina based on synthetic diagrams shows that the narrowness of the RGB is indeed consistent with the combined effects of the star formation and chemical enrichment history (Rizzi et al. 2003). The presence of a wide range in metallicity is confirmed by spectroscopy (Koch et al. 2004).

Figure 1 (right panel) shows the color-magnitude diagram of Sculptor. Sculptor is an "old" dwarf spheroidal galaxy lacking a significant star formation in the last 5 Gyr. It shows a particularly strong radial variation in the HB morphology index, which is accompanied by a spatial gradient in the RGB stars in the outer regions (Harbeck et al. 2001 Tolstoy et al. 2004 Babusiaux et al. 2005). The composite nature of the RGB (in particular the existence of a substantial metal-poor component) suggested by previous studies is not confirmed by recent photometry (Babusiaux et al. 2005). The presence of these gradients in both the HB morphology and the RGB implies that a significant 
metallicity gradient must be present (Rizzi et al. 2004). Using spectroscopic estimates of $[\mathrm{Fe} / \mathrm{H}]$ for RGB stars, Tolstoy et al. (2004) indeed find two components among red giant stars, with the metal-rich component more centrally concentrated than the metal-poor stars.

Similar CMD morphology gradients are seen in Sextans dSph (Harbeck et al. 2001 Lee et al. 2003). Our own synthetic CMD analysis suggests that in this case the stellar population gradient is driven by both metallicity and age variations. A hint for the origin of the changes in the stellar populations within Sextans dSph may also come from a comparison with internal kinematics. Indeed, Kleyna et al. (2004) associate a cold, inner stellar component having velocity dispersion close to zero with the change in the stellar populations near the center.

Other recent studies of old, metal-poor dwarf spheroidals include Draco, Ursa Minor, and Cetus (Aparicio et al. 2001, Carrera et al. 2002, Wyse et al. 2002, Sarajedini et al. 2002). In Draco and Ursa Minor, the metallicity distribution is narrow yet with a measurable spread and slightly different shapes indicating different star formation histories (Bellazzini et al. 2002).

\subsection{An extended star formation in old dwarf spheroidals ?}

One interesting result of wide-field observations is that star formation may have continued at a low rate up to a recent (a few Gyr) epoch even in predominantly old dwarf spheroidals. In the CMD of Sextans, Ursa Minor, and Sculptor (see Fig. 11), a "plume" of stars is obviously present above the main-sequence turnoff. If those stars are main sequence stars, their presence implies a modest rate of star formation up to $\sim 2$ Gyr ago, after the bulk of stars was formed at old epochs. However, the possibility cannot be ruled out that these stars are "blue stragglers" like those found in globular clusters, which are likely to be the evolved products of mass transfer in old binary systems (see Lee et al. 2003). The recent studies of Ursa Minor and Sextans conclude that the "blue plume" is more likely to be made of old GC-like "blue stragglers" rather than intermediate-age normal main-sequence stars (Carrera et al.2002 Lee et al.2003). A similar interpretation is proposed for the "blue plume" stars in Sculptor (Rizzi et al. 2004), while a different conclusion is reached by Aparicio et al. (2001) for Draco, where an analysis of the red clump and subgiant branch stars supports the existence of an intermediate age population, up to 2-3 Gyr ago. These different interpretations obviously affect our picture of early star formation in old dwarf spheroidals and their relation to the reionization era (see Grebel \& Gallagher 2004).

\subsection{Evolved stellar populations}

The intermediate-mass stars on the extended asymptotic giant branch (AGB), in particular the carbon-rich $(\mathrm{C})$ stars, are important tracers of intermediate-age populations in dwarf galaxies (for a review of AGB stars in Local Group galaxies, see Groenewegen 2004). Because of their high luminosities and distinctive spectral features, $\mathrm{C}$ stars are relatively easy to identify on the basis of narrow-band and near-infrared colors. Carbon star surveys have been conducted by different groups (e.g., Kerschbaum et al. 2004, Battinelli \& Demers 2005. Harbeck et al. 2004). The formation of C stars is both a function of the star formation history and metallicity. Because of this dependence on metallicity, not only is the ratio between carbon-rich and oxygen-rich cool giants, $\mathrm{C} / \mathrm{M}$, globally a function of galaxy metallicity (see, e.g., Battinelli \& Demers 2005, and references therein) but also it can be used to map local abundance variations across galaxies. New models including revised effects of variable opacity in carbon-rich stars are now able to better reproduce the observed distribution of luminous AGB stars in near-infrared CMDs (Marigo 2002). 
Since even a few luminous AGB stars can significantly contribute to the integrated light of galaxies, studying the behavior of AGB stars in nearby dwarfs represents an important step towards a successful intepretation of the spectra of distant dwarf galaxies. Evolutionary population synthesis models focusing on intermediate-age populations and their contribution to the integrated light have been presented by Mouhcine \& Lançon (2003).

The cool AGB as well as the RGB stars are best observed in the near-infrared, where bolometric corrections to intrinsic luminosities and effective temperatures are smaller, making interpretation of the observations on the basis of stellar evolution models more reliable. The optical-near infrared colors of RGB stars are more sensitive to metallicity than optical (or infrared) colors alone, in particular for old, metal-poor systems. This alleviates the age-metallicity degeneracy and allows an improved estimate of both mean metallicities and metallicity distributions (Babusiaux et al. 2005. Gullieuszik et al., this conference). If we exclude the Sagittarius dSph, which is close enough to be studied with the 2MASS database (e.g., Cole 2001), the sensitivity and small size of near-infrared instrumentation have limited the number of studies to very few dwarf spheroidals (Menzies et al. 2002 Pietrzyński et al. 2003. Babusiaux et al. 2005). Ongoing wide-area studies of dSph galaxies by our group as well as other teams aim at filling this gap, especially using the new wide-field instruments.

\subsection{Variable stars as stellar population tracers}

Pulsating variable stars have been studied in dSph galaxies for many years (Mateo 1998). Besides providing independent distance estimates to the galaxies, variable stars can trace the presence and properties of different stellar generations. In particular, RR Lyrae variables originate from the oldest stars ( $\gtrsim 10 \mathrm{Gyr}$ ), so that an old stellar population can be detected even when the data are too shallow or confusion-limited to reach the main sequence turnoff of low-mass stars. Measurements of RR Lyrae metallicity based on their pulsational properties (period and amplitude of the light curve) help us to reconstruct the metal enrichment history of dwarf spheroidals. In addition, the radial distribution of variable stars in different classes can be used to map the gradients in their parent stellar populations (e.g., Gallart et al. 2004).

Several recent studies have addressed the properties of variable stars in $\mathrm{dSph}$ galaxies (e.g., Leo I: Held et al. 2001] Fornax: Bersier \& Wood 2002, Poretti et al. 2005, in prep.; Carina: Dall'Ora et al. 2003. Draco: Bonanos et al. 2004 Phoenix: Gallart et al. 2004] and references therein). The RR Lyrae in most dSph galaxies appear to be intermediate between those in Oosterhoff type I and type II globular clusters (e.g., Dall'Ora et al. 2003), a characteristic shared by the globular clusters belonging to Fornax dSph (Mackey \& Gilmore 2003).

Other classes of variable stars bear information on the stellar content of local dwarfs. An interesting class is Anomalous Cepheids, which are found numerous in dSph galaxies, where their number per unit luminosity seems to be correlated to the galaxy metallicity and luminosity (e.g., Pritzl et al. 2005). That Anomalous Cepheids are related to the short-period Classical Cepheids found in (dwarf) irregular galaxies is still a matter of debate (Dolphin 2002 Baldacci et al. 2004, Marconi et al. 2004 Gallart et al. 2004] Pritzl et al. 2005). As such, the Anomalous Cepheid would trace the intermediate-age populations rather than the old component.

\subsection{Modeling the star formation histories}

Presently, the main challenge is to interpret observational facts in terms of galaxy evolution and understand the chemical enrichment and star formation histories of dSph

galaxies (see, e.g., Gallagher \& Grebel 2004). Disentangling the age and metallicity vari- 
ations of the stellar populations is a complex task that models based on evolutionary tracks and synthetic color-magnitude diagrams have recently undertaken (Hernandez et al. 2000, Ikuta \& Arimoto 2002 Dolphin 2002] Rizzi et al. 2004 Pont et al. 2004). While in many cases the models assume a metal enrichment law, some chemical evolution models have started to shed some on the interplay of star formation and chemical evolution of dwarf spheroidal galaxies (e.g., Ikuta \& Arimoto 2002 Carigi et al. 2002 Lanfranchi \& Matteucci 2004). For example, in the Lanfranchi \& Matteucci (2004) models, the metallicity distributions of stars in dSph galaxies are predicted using the star formation histories derived from the CMDs together with physical constraints such as the mass and gas content, and the observed abundance patterns from high-resolution spectroscopy (e.g., Tolstoy et al. 2003, Shetrone 2004). The overall properties of dwarf spheroidals imply very low star formation rates and a high efficiency of galactic winds in order to reproduce the observed gas fraction and abundance ratios, a conclusion on which the different models mostly agree.

Environmental factors, such as interaction with the Galaxy and M 31, may also influence the evolution of dSph galaxies. In the case of the Milky Way satellites, our understanding of the relationship between history of star formation and orbital motions can benefit from recent analyses of proper motions. For Fornax, the work of Dinescu et al. (2004) suggests that the termination of star formation about 200 Myr ago indicated by color-magnitude diagrams coincides with the time when Fornax crossed the Magellanic Plane. However, in the case of Carina, the history of star formation is not explained by passages through perigalacticon and the Galactic disk - the interval between multiple passages being too short to explain the main star formation episode about 6-7 Gyr ago (Piatek et al. 2003). Thus, while interaction with the Milky Way is suggested to be able to trigger or stop star formation (by gas removal), it is certainly not the only driving factor of their star formation histories.

I wish to thank Ivo Saviane, Yazan Momany, Luca Rizzi, Marco Gullieuszik, and Gianpaolo Bertelli, my collaborators on stellar population research, and the organizers of this enjoyable meeting. The search for variable stars in local dwarfs is done in collaboration with teams led by G. Clementini, E. Poretti, H. Smith, and M. Catelan.

\section{References}

Aparicio, A., Carrera, R., \& Martínez-Delgado, D. 2001, AJ, 122, 2524

Babusiaux, C., Gilmore, G., \& Irwin, M. 2005, MNRAS, 331

Baldacci, L., Clementini, G., Held, E. V., Marconi, M., \& Rizzi, L. 2004, in JENAM 2003 Minisymposium on "Asteroseismology and Stellar Evolution", Communications in Asteroseismology 145,32

Battinelli, P., \& Demers, S. 2005, A\&A, 434, 657

Bellazzini, M., Ferraro, F. R., Origlia, L., Pancino, E., Monaco, L., \& Oliva, E. 2002, AJ, 124, 3222

Bersier, D., \& Wood, P. R. 2002, AJ, 123, 840

Bonanos, A. Z., Stanek, K. Z., Szentgyorgyi, A. H., Sasselov, D. D., \& Bakos, G. Á. 2004, AJ, 127,861

Carigi, L., Hernandez, X., \& Gilmore, G. 2002, MNRAS, 334, 117

Carrera, R., Aparicio, A., Martínez-Delgado, D., \& Alonso-García, J. 2002, AJ, 123, 3199

Cole, A. A. 2001, ApJL, 559, L17

Da Costa, G. S. 1998, in "Stellar astrophysics for the Local Group : VIII Canary Islands Winter School of Astrophysics", Eds. A. Aparicio, A. Herrero, and F. Sanchez, Cambridge, New York : Cambridge University Press, p.351

Dall'Ora, M., et al. 2003, AJ, 126, 197 
Dinescu, D. I., Keeney, B. A., Majewski, S. R., \& Girard, T. M. 2004, AJ, 128, 687

Dolphin, A. E. 2002, MNRAS, 332, 91

Gallagher, J. S., \& Grebel, E. K. 2004, in "Origin and Evolution of the Elements', Carnegie Observatories Centennial Symposia, Cambridge University Press, Eds. A. McWilliam and M. Rauch

Gallart, C., Aparicio, A., Freedman, W. L., Madore, B. F., Martínez-Delgado, D., \& Stetson, P. B. 2004, AJ, 127, 1486

Grebel, E. K. 2000. in "Star formation from the small to the large scale", ESLAB Symp. 33, Eds. F. Favata, A. Kaas, and A. Wilson, The Netherlands: European Space Agency, p. 87

Grebel, E. K., \& Gallagher, J. S. 2004, ApJL, 610, L89

Groenewegen M. 2004, in ESO Workshop "Planetary Nebulae beyond the Milky Way" (arXiv:astro-ph/0407282)

Harbeck, D., et al. 2001, AJ, 122, 3092

Harbeck, D., Gallagher, J. S., \& Grebel, E. K. 2004, AJ, 127, 2711

Held, E. V., Saviane, I., Momany, Y., \& Carraro, G. 2000, ApJL, 530, L85

Held, E. V., Clementini, G., Rizzi, L., Momany, Y., Saviane, I., \& Di Fabrizio, L. 2001, ApJL, 562 , L39

Hernandez, X., Gilmore, G., \& Valls-Gabaud, D. 2000, MNRAS, 317, 831

Ikuta, C., \& Arimoto, N. 2002, A\& A, 391, 55

Kerschbaum, F., Nowotny, W., Olofsson, H., \& Schwarz, H. E. 2004, A\& A, 427, 613

Kleyna, J. T., Wilkinson, M. I., Evans, N. W., \& Gilmore, G. 2004, MNRAS, 354, L66

Koch, A., Grebel, E. K., Harbeck, D., Wilkinson, M. I., Kleyna, J. T., Gilmore, G. F., Wyse, R. F. G., \& Evans, N. W. 2004, in "Chemical Abundances and Mixing in Stars", eds. L. Pasquini \& S. Randich, in press (arXiv:astro-ph/0410435)

Lanfranchi, G. A., \& Matteucci, F. 2004, MNRAS, 351, 1338

Lee, M. G., et al. 2003, $A J, 126,2840$

Mackey, A. D., \& Gilmore, G. F. 2003, MNRAS, 345, 747

Marconi, M., Fiorentino, G., \& Caputo, F. 2004, A\&A, 417, 1101

Marigo, P. 2002, $A \mathscr{E} A, 387,507$

Mateo, M. L. 1998, ARA\&A, 36, 435

Menzies, J., Feast, M., Tanabé, T., Whitelock, P., \& Nakada, Y. 2002, MNRAS, 335, 923

Monelli, M., et al. 2003, AJ, 126, 218

Mouhcine, M., \& Lançon, A. 2003, MNRAS, 338, 572

Piatek, S., Pryor, C., Olszewski, E. W., Harris, H. C., Mateo, M., Minniti, D., \& Tinney, C. G. 2003, AJ, 126, 2346

Pietrzyński, G., Gieren, W., \& Udalski, A. 2003, AJ, 125, 2494

Pont, F., Zinn, R., Gallart, C., Hardy, E., \& Winnick, R. 2004, AJ, 127, 840

Pritzl, B. J., Armandroff, T. E., Jacoby, G. H., \& Da Costa, G. S. 2005, AJ, 129, 2232

Rizzi, L., Held, E. V., Bertelli, G., \& Saviane, I. 2003, ApJL, 589, L85

Rizzi, L., Held, E. V., Bertelli, G., \& Saviane, I. 2004, MmSAI, 75, 110

Sarajedini, A., et al. 2002, ApJ, 567, 915

Saviane, I., Held, E. V., \& Bertelli, G. 2000, A $\xi A$, 355, 56

Shetrone M. D. 2004, in "Origin and Evolution of the Elements', Carnegie Observatories Centennial Symposia, Cambridge University Press, Eds. A. McWilliam and M. Rauch, p. 220

Tolstoy, E. 2003, in "A Decade of Hubble Space Telescope Science", Eds. M. Livio, K. Noll, M. Stiavelli STScI Symp. Series Vol. 14, Cambridge, UK: Cambridge University Press, p. 128

Tolstoy, E., Venn, K. A., Shetrone, M., Primas, F., Hill, V., Kaufer, A., \& Szeifert, T. 2003, AJ, 125,707

Tolstoy, E., et al. 2004, ApJL, 617, L119

van den Bergh, S. 2000, "The galaxies of the Local Group", Cambridge Astrophysics Series vol. 35 (Cambridge, UK: Cambridge University Press)

Wyse, R. F. G., Gilmore, G., Houdashelt, M. L., Feltzing, S., Hebb, L., Gallagher, J. S., \& Smecker-Hane, T. A. 2002, NewA, 7, 395 